\title{
Phase I study of ipilimumab in phased combination with paclitaxel and carboplatin in Japanese patients with non-small-cell lung cancer
}

\author{
Hidehito Horinouchi $^{1}$ - Noboru Yamamoto ${ }^{1}$ - Yutaka Fujiwara ${ }^{1}$ - Ikuo Sekine ${ }^{1}$. \\ Hiroshi Nokihara $^{1}$ - Kaoru Kubota ${ }^{1}$ - Shintaro Kanda ${ }^{1}$ - Shigehiro Yagishita ${ }^{1}$. \\ Hiroshi Wakui $^{1}$ - Satoru Kitazono ${ }^{1}$ - Hidenori Mizugaki ${ }^{1}$ • Takuto Tokudome ${ }^{2}$. \\ Tomohide Tamura ${ }^{1}$
}

Received: 17 February 2015 / Accepted: 15 April 2015 /Published online: 1 May 2015

(C) The Author(s) 2015. This article is published with open access at Springerlink.com

Summary Background Ipilimumab is an antibody that targets the cytotoxic T-lymphocyte antigen-4 to potentiate an antitumor response. Adding ipilimumab $10 \mathrm{mg} / \mathrm{kg}$ to paclitaxel (PTX) and carboplatin (CBDCA) in a phased schedule improved progression-free survival in a phase II non-small-cell lung cancer (NSCLC) study. Methods This dose-escalating, phase I study was designed to identify the recommended dose of ipilimumab (3 or $10 \mathrm{mg} / \mathrm{kg}$ ) by evaluating dose-limiting toxicity (DLT; Cycles 3 and 4) in phased combination with PTX $\left(175 \mathrm{mg} / \mathrm{m}^{2}\right)$ and CBDCA (area under the curve $\left.=6\right)$ in Japanese patients with advanced NSCLC. Treatment was administered intravenously every 3 weeks initially, followed by some eligible patients receiving maintenance ipilimumab once every 12 weeks. Additional endpoints included safety, tumor response, pharmacokinetics, and immunogenicity. Results Fifteen patients were enrolled and 12 received ipilimumab $(n=6,3 \mathrm{mg} / \mathrm{kg} ; n=6,10 \mathrm{mg} / \mathrm{kg})$ in combination with PTX and CBDCA. DLTs occurred in 2 patients (ipilimumab $3 \mathrm{mg} / \mathrm{kg}$ ) and 1 patient (ipilimumab $10 \mathrm{mg} / \mathrm{kg}$ ). The most common grade 3/4 adverse events (AEs) were decreased hemoglobin, leukopenia, and neutropenia. The most common immune-related AEs affected the skin, gastrointestinal, and nervous system. The safety profile was similar in both cohorts. Three patients in each cohort achieved a partial response. The pharmacokinetic (PK)

Hidehito Horinouchi

hhorinou@ncc.go.jp

1 Department of Thoracic Oncology, National Cancer Center Hospital, Tsukiji 5-1-1, Chuo-ku, Tokyo 104-0045, Japan

2 Bristol-Myers K.K., Clinical Research, R\&D, 6-5-1 Nishishinjyuku, Shinjyuku-ku, Tokyo 163-1328, Japan profile of ipilimumab in Japanese patients was similar to that observed in previous studies in non-Japanese patients. Conclusions The recommended dose of ipilimumab in phased combination with PTX and CBDCA in Japanese patients with NSCLC was identified as $10 \mathrm{mg} / \mathrm{kg}$. The safety profile was consistent with the previously defined AE profile.

Keywords Ipilimumab · Paclitaxel $\cdot$ Carboplatin

Non-small-cell lung cancer · Phase I study · Japanese patients

\section{Introduction}

Patients with newly diagnosed non-small-cell lung cancer (NSCLC) typically present with locally advanced stage III or metastatic stage IV disease. Platinum-based chemotherapy combinations, the current standard of care for this patient population, offer responses of between 30 and $60 \%$, but survival benefit is limited (median overall survival of approximately 9 months), and new treatment options are needed [1]. One of the most promising developments in the search for novel treatments has been the advent of immune-checkpoint inhibitors that harness the power of the patient's own immune system to fight cancer [2]. Ipilimumab is a fully human, monoclonal antibody that targets the immune-checkpoint cytotoxic T-lymphocyte antigen-4 (CTLA-4) to potentiate T-cell-mediated antitumor response [3]. Ipilimumab is approved as either first- or secondline therapy in over 40 countries for the treatment of unresectable or metastatic melanoma, but is not currently approved in Japan $[4,5]$. The potential use of ipilimumab in the treatment of other tumor types is an area of intense investigation.

Recently, ipilimumab $10 \mathrm{mg} / \mathrm{kg}$ in combination with paclitaxel (PTX) and carboplatin (CBDCA) was evaluated in 
patients with previously untreated, advanced NSCLC in a randomized, multicenter, double blind, phase II study [6]. In this study, patients received ipilimumab in a phased or concurrent regimen. Patients treated with the phased regimen received two doses of placebo plus PTX/CBDCA, followed by four doses of ipilimumab plus PTX/CBDCA; those treated with the concurrent regimen received four doses of ipilimumab plus PTX/CBDCA, followed by two doses of placebo plus PTX/CBDCA. Treatment was administered intravenously every 3 weeks for $\leq 18$ weeks (induction). Eligible patients continued ipilimumab or placebo every 12 weeks as maintenance therapy. The addition of ipilimumab to PTX/CBDCA in a phased schedule significantly increased progression-free survival (PFS) compared with PTX/CBDCA alone. No statistically significant difference was observed between concurrent ipilimumab and PTX/CBDCA versus PTX/CBDCA alone. Ipilimumab in combination with PTX/CBDCA was well tolerated. Taken together, these results suggest that ipilimumab in combination with PTX/CBDCA on a phased schedule was safe and active in this patient population.

The primary objective of this open-label, dose-escalation, phase I study (CA184-113, ClinicalTrials.gov: NCT01165216) was to establish the recommended dose of ipilimumab in a phased combination regimen with PTX/ CBDCA in Japanese patients with advanced or metastatic NSCLC. Safety, tumor response, pharmacokinetics, and immunogenicity were also assessed.

\section{Methods}

\section{Patients}

Japanese patients of either sex were eligible to participate in this study if they had histologically- or cytologicallydocumented stage IIIB NSCLC and were not candidates for definitive thoracic radiotherapy, stage IV NSCLC, or recurrent disease following radiotherapy and/or surgery. Eligible patients were required to have adequate bone marrow function (hemoglobin $\geq 9.0 \mathrm{~g} / \mathrm{dL}$, absolute neutrophil count $[\mathrm{ANC}] \geq$ $1500 / \mathrm{mm}^{3}$, and platelet count $\geq 100,000 / \mathrm{mm}^{3}$ ), liver function (total bilirubin $\leq 2.0$ times the upper limit of normal [x ULN], aspartate aminotransferase $[\mathrm{AST}] \leq 2.5 \times \mathrm{ULN}$, alanine aminotransferase $[\mathrm{ALT}] \leq 2.5 \times \mathrm{ULN}$ ), and renal function (calculated creatinine clearance based on Cockcroft and Gault formula $\geq 50 \mathrm{~mL} / \mathrm{min}$ ). Other inclusion criteria included age of at least 20 years; no prior chemotherapy, hormonal therapy, immunotherapy or targeted therapy for NSCLC; a life expectancy of at least 3 months; an Eastern Cooperative Oncology Group (ECOG) performance status of 0 or 1; and the ability and willingness to comply with visits and procedures as specified in the protocol (patients were admitted to the hospital from at least Day 1 to Day 22 of the third treatment cycle).
Patients were ineligible to participate in this study if they had symptomatic brain metastases or brain metastases requiring medication, a history of autoimmune disease or motor neuropathy of autoimmune origin, peripheral neuropathy of grade 2 or higher, diarrhea of grade 2 or higher, current or a history of gastrointestinal perforations, and chronic use of immunosuppressants and/or systemic corticosteroids.

\section{Study oversight}

This study was sponsored by Bristol-Myers K.K. All procedures performed in studies involving human participants were in accordance with the ethical standards of the institutional and/or national research committee and with the 1964 Helsinki declaration and its later amendments or comparable ethical standards. The study protocol was approved by the Institutional Review Board and research was conducted in accordance with the standards specified by Article 14 Paragraph 3 and Article 80-2 of the Pharmaceutical Affairs Law, and Good Clinical Practice, as defined by the Ministerial Ordinance Concerning the Standards for the Implementation of Clinical Studies on Pharmaceutical Products and concerning notifications. All study participants provided written informed consent prior to enrollment.

\section{Study design and treatment}

In this open-label, dose-escalation, phase I study, patients with advanced or metastatic NSCLC were treated with ipilimumab (3 or $10 \mathrm{mg} / \mathrm{kg}$ ) in combination with PTX $\left(175 \mathrm{mg} / \mathrm{m}^{2}\right)$ and CBDCA (area under the curve $[\mathrm{AUC}]=6$ ). The treatment schedule was divided into two treatment periods (Fig. 1). During the first treatment period, patients received phased ipilimumab, which consisted of two cycles of PTX/CBDCA every 3 weeks ( $\mathrm{q} 3 \mathrm{wks}$ ) followed by four cycles of ipilimumab plus PTX/CBDCA q3wks. During the second treatment period, patients without disease progression received ipilimumab alone once every 12 weeks (q12wks) as maintenance treatment. To be eligible for maintenance treatment, patients were required to meet core safety criteria and have adequate bone marrow function (ANC $>1500 / \mathrm{mm}^{3}$ and platelet count $>100$,

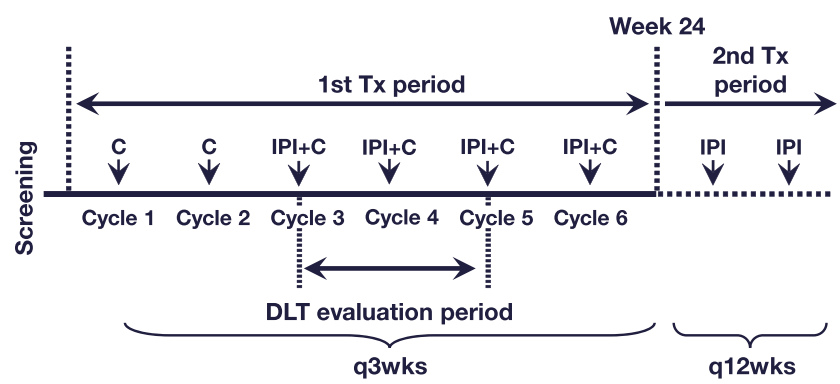

Fig. 1 Schema for study CA184-113. C, paclitaxel/carboplatin; DLT, dose-limiting toxicity; IPI, ipilimumab; Tx, treatment; q3wks, every 3 weeks; q12wks, every 12 weeks 
$000 / \mathrm{mm}^{3}$ ). Patients were enrolled in successive cohorts of 3 to 6 patients using a standard ' $3+3$ ' design. The recommended dose was defined as the highest dose at which not more than 2 out of 6 patients experienced a dose-limiting toxicity (DLT), taking into consideration the profile of DLTs and the frequency and severity of toxicities after the DLT evaluation period. The final recommended dose was agreed upon by the Sponsor and principal investigator. Patients received ipilimumab at $3 \mathrm{mg} / \mathrm{kg}$ (Cohort A) or $10 \mathrm{mg} / \mathrm{kg}$ (Cohort B).

\section{Study endpoints and assessments}

The primary objective of this study was to identify the recommended dose of ipilimumab in a phased combination regimen with PTX/CBDCA in Japanese patients with advanced or metastatic NSCLC. The recommended dose was defined as the highest dose at which not more than 2 out of 6 patients had DLT during the first two cycles after ipilimumab treatment (i.e., from Day 1 at Cycle 3 to Day 21 at Cycle 4). Doselimiting toxicities included any of the following: grade 4 ANC decrease lasting for 7 days or more; febrile neutropenia lasting more than 3 days; grade 4 platelet count decrease; grade 3 platelet count decrease requiring transfusion; grade 3 or higher nausea, vomiting, and/or diarrhea despite the use of adequate/maximal medical intervention; grade 3 or higher AST/ALT levels not resolved to grade 2 or less within 2 weeks of onset; grade 3 or higher rash not resolved to grade 2 or less within 2 weeks of onset; and any grade 3 or higher nonhematological toxicity (except grade 3 fatigue, grade 3 asthenia, grade 3 transient arthralgia/myalgia, and grade 3 transient abnormal electrolyte).

Secondary objectives were safety and tolerability, tumor response, and pharmacokinetics (PK) of ipilimumab. Safety and tolerability was defined as the incidence of AEs occurring throughout the study period. These assessments were based on the National Cancer Institute Common Terminology Criteria for Adverse Events (NCI-CTCAE) version 3.0 [7]. Tumor response was defined as the best overall response rate (BORR). Responses were based on Response Evaluation Criteria in Solid Tumors (RECIST) version 1.1 [8]. Blood serum samples for assessing PK of ipilimumab were drawn at the following time points: Cycle 3 (Day 1, 2, 3, 8, and 15), Cycle 4 (Day 1), Cycle 5 (Day 1), Cycle 6 (Day 1), Week 24 and subsequent cycles, and off treatment. Quantification of ipilimumab in serum was performed using a validated enzyme linked immunosorbent assay (ELISA). Ipilimumab PKs were derived from serum concentration-versus-time data. Pharmacokinetic parameters included peak plasma concentration $\left(\mathrm{C}_{\max }\right)$, area under the concentration-versus-time curve from time zero to Day $21\left(\mathrm{AUC}_{0-21 \mathrm{~d}}\right)$, time of maximum observed serum concentration $\left(\mathrm{T}_{\max }\right)$, serum half-life (T-HALF), clearance $(\mathrm{CL})$, and volume of distribution at steady-state
(Vss). Geometric mean and coefficient of variation were calculated for $\mathrm{C}_{\max }, \mathrm{AUC}_{0-21 \mathrm{~d}}, \mathrm{CL}$, and Vss. Mean and standard deviation were calculated for T-HALF. Median, minimum and maximum were calculated for $\mathrm{T}_{\max }$.

Immunogenicity was evaluated as an exploratory endpoint. Blood samples were evaluated for the presence of antibodies to ipilimumab (human anti-human antibodies [HAHA]). Blood samples were drawn pre-dose on Day 1 of the third cycle and prior to each subsequent dose of ipilimumab, off treatment, and at first follow-up in case of discontinuation before Week 24. The detection of HAHA was performed using a validated bridging electrochemiluminescence immunoassay, which provided a semi-quantitative assessment of immunogenicity for HAHA using preestablished cut-points.

\section{Results}

\section{Patients and treatment}

Fifteen patients with advanced or metastatic NSCLC were enrolled and treated at the National Cancer Center Hospital in Tokyo, Japan between September 2010 and June 2013; patient characteristics at baseline are provided in Table 1. Eight patients were enrolled in Cohort A (ipilimumab $3 \mathrm{mg} / \mathrm{kg}$ ), and 7 patients were enrolled in Cohort B (ipilimumab $10 \mathrm{mg} / \mathrm{kg}$ ). Six patients in each study cohort received ipilimumab treatment. Three patients were

Table 1 Patient characteristics

\begin{tabular}{|c|c|c|c|}
\hline Characteristic & $\begin{array}{l}\text { Cohort A } \\
(n=8) \\
\text { Ipi } \\
3 \mathrm{mg} / \mathrm{kg}\end{array}$ & $\begin{array}{l}\text { Cohort B } \\
(n=7) \\
\text { Ipi } \\
10 \mathrm{mg} / \mathrm{kg}\end{array}$ & $\begin{array}{l}\text { Total } \\
\left(N=15^{\mathrm{a}}\right)\end{array}$ \\
\hline Number of ipilimumab-treated patients & 6 & 6 & 12 \\
\hline \multicolumn{4}{|l|}{ Sex, n (\%) } \\
\hline Male & $6(75.0)$ & $6(85.7)$ & $12(80.0)$ \\
\hline Female & $2(25.0)$ & $1(14.3)$ & $3(20.0)$ \\
\hline \multicolumn{4}{|l|}{ Age, years } \\
\hline Median & 61.5 & 61.0 & 61.0 \\
\hline Range & $27-65$ & $40-70$ & $27-70$ \\
\hline \multicolumn{4}{|l|}{ Disease status, $\mathrm{n}(\%)$} \\
\hline Stage IV & $6(75.0)$ & $5(71.4)$ & $11(73.3)$ \\
\hline Recurrent & $2(25.0)$ & $2(28.6)$ & $4(26.7)$ \\
\hline \multicolumn{4}{|l|}{ Tumor type, n (\%) } \\
\hline Adenocarcinoma & $8(100.0)$ & $6(85.7)$ & $14(93.3)$ \\
\hline Squamous cell carcinoma & $0(0.0)$ & $1(14.3)$ & $1(6.7)$ \\
\hline
\end{tabular}

Ipi ipilimumab

${ }^{a}$ Including patients who were discontinued before receiving ipilimumab due to intolerable toxicity associated with chemotherapy $(n=2$, Cohort $\mathrm{A})$ and withdrawal of informed consent $(n=1$, Cohort B) 
discontinued prior to receiving ipilimumab. The reasons for discontinuation were intolerable toxicity $(n=2$, Cohort $\mathrm{A})$ and withdrawal of informed consent $(n=1$, Cohort $\mathrm{B})$. The median age was similar in the two dose cohorts and the majority of patients were male with stage IV disease. All patients had adenocarcinoma, with the exception of one patient in Cohort $\mathrm{B}$ who had squamous cell carcinoma.

Treatment and dosing information is provided in Table 2. The majority of patients required a dose delay of study drug ( $n=11 / 12$, ipilimumab; $n=12 / 15$, PTX/CBDCA). Two patients in Cohort A (ipilimumab $3 \mathrm{mg} / \mathrm{kg}$ ) and one patient in Cohort B (ipilimumab $10 \mathrm{mg} / \mathrm{kg}$ ) required a chemotherapy dose reduction, which occurred during the fifth or sixth treatment cycle. One patient in Cohort B received a maintenance dose of ipilimumab, and all patients were off treatment at the end of the study. For the total study population, the reasons for study discontinuation $(n=15)$ were study drug toxicity $(60 \%)$, disease progression (26.7\%), and discontinuation at the patient's request (13.3\%). In Cohort A (ipilimumab $3 \mathrm{mg} / \mathrm{kg}, n=$ 8), 6 patients discontinued due to study drug toxicity $(75 \%), 1$ patient discontinued due to disease progression $(12.5 \%)$, and 1 patient requested to discontinue (12.5\%). In Cohort B (ipilimumab $10 \mathrm{mg} / \mathrm{kg}, n=7$ ), 3 patients discontinued due to study drug toxicity $(42.9 \%), 3$ patients discontinued due to disease progression (42.9\%), and 1 patient requested to discontinue (14.3\%).

Table 2 Treatment and number of doses that patients received

\begin{tabular}{clll}
\hline & $\begin{array}{l}\text { Cohort A } \\
(n=8) \\
\text { Ipi 3 mg/kg }\end{array}$ & $\begin{array}{l}\text { Cohort B } \\
(n=7) \\
\text { Ipi 10 mg/kg }\end{array}$ & $\begin{array}{l}\text { Total } \\
(N=15)\end{array}$ \\
\hline $\begin{array}{l}\text { Number of ipilimumab-treated } \\
\text { patients, } \mathrm{n}\end{array}$ & 6 & 6 & 12 \\
$\begin{array}{c}\text { Number of doses, } \mathrm{n} \\
\text { Ipilimumab }\end{array}$ & & & \\
0 & $2(25.0)$ & $1(14.3)$ & $3(20.0)$ \\
1 & $0(0)$ & $0(0)$ & $0(0)$ \\
2 & $3(37.5)$ & $1(14.3)$ & $4(26.7)$ \\
3 & $0(0)$ & $2(28.6)$ & $2(13.3)$ \\
4 & $3(37.5)$ & $2(28.6)$ & $5(33.3)$ \\
5 (maintenance dose) & $0(0)$ & $1(14.3)$ & $1(6.7)$ \\
6 (maintenance dose) & $0(0)$ & $0(0)$ & $0(0)$ \\
Paclitaxel/carboplatin & & & \\
0 & $0(0)$ & $0(0)$ & $0(0)$ \\
1 & $2(25.0)$ & $0(0)$ & $2(13.3)$ \\
2 & $0(0)$ & $1(14.3)$ & $1(6.7)$ \\
3 & $0(0)$ & $0(0)$ & $0(0)$ \\
4 & $3(37.5)$ & $1(14.3)$ & $4(26.7)$ \\
5 & $0(0)$ & $4(57.1)$ & $4(26.7)$ \\
6 & $3(37.5)$ & $1(14.3)$ & $4(26.7)$ \\
\hline
\end{tabular}

Ipi ipilimumab

\section{Safety data}

In Cohort A (ipilimumab $3 \mathrm{mg} / \mathrm{kg}$ ), DLT was reported in 2 of 6 patients. One patient experienced grade 3 febrile neutropenia and grade 4 increased amylase after 4 doses of chemotherapy and 2 doses of ipilimumab. The febrile neutropenia resolved after treatment with granulocyte colony-stimulating factor (G-CSF) and antibiotics; the increased amylase required no treatment. Another patient experienced grade 4 thrombocytopenia after 4 doses of chemotherapy and 2 doses of ipilimumab. The thrombocytopenia resolved after a platelet transfusion. In Cohort B (ipilimumab $10 \mathrm{mg} / \mathrm{kg}$ ), DLT was reported in 1 of 6 patients. This patient experienced grade 3 enterocolitis, grade 3 increased total bilirubin, and grade 4 increased lipase after 4 doses of chemotherapy and two doses of ipilimumab. The enterocolitis and increased total bilirubin resolved with treatment, which included a high-dose steroid (prednisone), levofloxacin, meropenem, and parenteral nutrition; and the increase lipase resolved without treatment.

A summary of treatment-related adverse events (AEs) in patients who received PTX/CBDCA and at least one dose of ipilimumab is shown in Table 3. The most common grade 3/4 AEs (occurring in 3 or more patients) were neutropenia ( $n=6$, Cohort $\mathrm{A} ; n=6$, Cohort $\mathrm{B})$, decreased hemoglobin $(n=4$, Cohort $\mathrm{B})$, and leukopenia $(n=3$, Cohort $\mathrm{A} ; n=1$, Cohort $\mathrm{B})$. The most frequent lower-grade (1/2) AEs that occurred in greater than $20 \%$ of patients in either cohort included decreased appetite ( $n=4$, cohort $\mathrm{A} ; n=5$, cohort $\mathrm{B})$, arthralgia $(n=3$, cohort A, $n=5$, cohort $\mathrm{B})$, rash ( $n=5$, cohort $\mathrm{A} ; n=3$, cohort $\mathrm{B})$, nausea $(n=4$, cohort $\mathrm{A} ; n=3$, cohort $\mathrm{B})$, prolonged QT ( $n=4$, cohort $\mathrm{A} ; n=2$, cohort $\mathrm{B})$, fatigue ( $n=2$, cohort $\mathrm{A}$; $n=4$, cohort B), and peripheral sensory neuropathy ( $n=1$, cohort $\mathrm{A} ; n=5$, cohort $\mathrm{B}$ ).

Table 4 provides a summary of immune-related adverse events (irAEs) in patients who received PTX/CBDCA and at least one dose of ipilimumab. The most commonly reported irAEs across the two study cohorts were grade $1 / 2$ toxicities affecting the skin and subcutaneous tissues $(n=5$, Cohort A; $n=6$, Cohort B), gastrointestinal tract $(n=4$, Cohort $\mathrm{A} ; n=1$, Cohort $\mathrm{B}$ ), and nervous system ( $n=3$, Cohort $\mathrm{A} ; n=5$, Cohort B). Two patients in Cohort A experienced a grade $3 / 4$ irAE $(n=1$, grade 3 adrenal insufficiency; $n=1$, grade 4 increase in amylase). The grade 3 adrenal insufficiency occurred on Day 159 , after 6 cycles of chemotherapy and 4 doses of ipilimumab. It was treated with oral prednisolone that was continued through to the last follow-up assessment due to unresolved decreases in both adrenocorticotropic hormone and cortisol. The grade 4 increase in amylase required no treatment. Two patients in Cohort B experienced a grade 3/4 irAE ( $n=1$, grade 3 enterocolitis, grade 3 increase in blood 
Table 3 Summary of treatment-related adverse events in patients who received PTX/CBDCA and at least one dose of ipilimumab

\begin{tabular}{|c|c|c|c|c|c|c|}
\hline \multirow[t]{2}{*}{ Toxicities $^{\mathrm{a}, \mathrm{b}}$} & \multicolumn{3}{|l|}{$\begin{array}{l}\text { Cohort A } \\
(n=6) \\
\text { Ipi } 3 \mathrm{mg} / \mathrm{kg}\end{array}$} & \multicolumn{3}{|l|}{$\begin{array}{l}\text { Cohort B } \\
(n=6) \\
\text { Ipi } 10 \mathrm{mg} / \mathrm{kg}\end{array}$} \\
\hline & Total (G1-4) & G3 & G4 & Total (G1-4) & G3 & G4 \\
\hline \multicolumn{7}{|l|}{ Hematological, n (\%) } \\
\hline Hemoglobin decreased & $0(0)$ & $0(0)$ & $0(0)$ & $4(66.7)$ & $4(66.7)$ & $0(0)$ \\
\hline Leukopenia & $3(50.0)$ & $3(50.0)$ & $0(0)$ & $1(16.7)$ & $1(16.7)$ & $0(0)$ \\
\hline Lymphopenia & $1(16.7)$ & $0(0)$ & $1(16.7)$ & $0(0)$ & $0(0)$ & $0(0)$ \\
\hline Neutropenia & $6(100.0)$ & $0(0)$ & $6(100.0)$ & $6(100.0)$ & $3(50.0)$ & $3(50.0)$ \\
\hline Febrile neutropenia & $1(16.7)$ & $1(16.7)$ & $0(0)$ & $0(0)$ & $0(0)$ & $0(0)$ \\
\hline Thrombocytopenia & $1(16.7)$ & $0(0)$ & $1(16.7)$ & $2(33.3)$ & $0(0)$ & $0(0)$ \\
\hline \multicolumn{7}{|l|}{ Gastrointestinal, n (\%) } \\
\hline Nausea & $4(66.7)$ & $0(0)$ & $0(0)$ & $3(50.0)$ & $0(0)$ & $0(0)$ \\
\hline Diarrhea & $3(50.0)$ & $0(0)$ & $0(0)$ & $1(16.7)$ & $0(0)$ & $0(0)$ \\
\hline Constipation & $2(33.3)$ & $0(0)$ & $0(0)$ & $2(33.3)$ & $0(0)$ & $0(0)$ \\
\hline Vomiting & $1(16.7)$ & $0(0)$ & $0(0)$ & $2(33.3)$ & $0(0)$ & $0(0)$ \\
\hline Enterocolitis & $0(0)$ & $0(0)$ & $0(0)$ & $1(16.7)$ & $1(16.7)$ & $0(0)$ \\
\hline \multicolumn{7}{|c|}{ Metabolism and nutrition disorders, $\mathrm{n}(\%)$} \\
\hline Decreased appetite & $4(66.7)$ & $0(0)$ & $0(0)$ & $5(83.3)$ & $0(0)$ & $0(0)$ \\
\hline Hypocalcaemia & $1(16.7)$ & $1(16.7)$ & $0(0)$ & $0(0)$ & $0(0)$ & $0(0)$ \\
\hline Hypomagnesaemia & $1(16.7)$ & $0(0)$ & $1(16.7)$ & $0(0)$ & $0(0)$ & $0(0)$ \\
\hline \multicolumn{7}{|l|}{ Dermatologic, n (\%) } \\
\hline Rash & $5(83.3)$ & $0(0)$ & $0(0)$ & $3(50.0)$ & $0(0)$ & $0(0)$ \\
\hline Alopecia & $1(16.7)$ & $0(0)$ & $0(0)$ & $2(33.3)$ & $0(0)$ & $0(0)$ \\
\hline Dry skin & $1(16.7)$ & $0(0)$ & $0(0)$ & $2(33.3)$ & $0(0)$ & $0(0)$ \\
\hline Pruritus & $0(0)$ & $0(0)$ & $0(0)$ & $2(33.3)$ & $0(0)$ & $0(0)$ \\
\hline \multicolumn{7}{|l|}{ Mediastinal disorders, $\mathrm{n}(\%)$} \\
\hline Hiccups & $0(0)$ & $0(0)$ & $0(0)$ & $2(33.3)$ & $0(0)$ & $0(0)$ \\
\hline Oropharyngeal pain & $2(33.3)$ & $0(0)$ & $0(0)$ & $2(33.3)$ & $0(0)$ & $0(0)$ \\
\hline \multicolumn{7}{|c|}{ Musculoskeletal and connective tissue disorders, $\mathrm{n}(\%)$} \\
\hline Arthralgia & $3(50.0)$ & $0(0)$ & $0(0)$ & $5(83.3)$ & $0(0)$ & $0(0)$ \\
\hline Peripheral sensory neuropathy & $1(16.7)$ & $0(0)$ & $0(0)$ & $5(83.3)$ & $0(0)$ & $0(0)$ \\
\hline \multicolumn{7}{|l|}{ Other, n (\%) } \\
\hline Blood sodium decreased & $0(0)$ & $0(0)$ & $0(0)$ & $1(16.7)$ & $1(16.7)$ & $0(0)$ \\
\hline GGT increased & $0(0)$ & $0(0)$ & $0(0)$ & $1(16.7)$ & $1(16.7)$ & $0(0)$ \\
\hline Hyponatremia & $1(16.7)$ & $1(16.7)$ & $0(0)$ & $1(16.7)$ & $1(16.7)$ & $0(0)$ \\
\hline Hypophosphatemia & $0(0)$ & $0(0)$ & $0(0)$ & $1(16.7)$ & $1(16.7)$ & $0(0)$ \\
\hline Lipase increased & $0(0)$ & $0(0)$ & $0(0)$ & $1(16.7)$ & $0(0)$ & $1(16.7)$ \\
\hline Amylase increased & $1(16.7)$ & $0(0)$ & $1(16.7)$ & $1(16.7)$ & $0(0)$ & $0(0)$ \\
\hline Blood bilirubin increased & $0(0)$ & $0(0)$ & $0(0)$ & $1(16.7)$ & $1(16.7)$ & $0(0)$ \\
\hline Adrenal insufficiency & $1(16.7)$ & $1(16.7)$ & $0(0)$ & $0(0)$ & $0(0)$ & $0(0)$ \\
\hline Prolonged QT & $4(66.7)$ & $0(0)$ & $0(0)$ & $2(33.3)$ & $0(0)$ & $0(0)$ \\
\hline Weight decrease & $3(50.0)$ & $0(0)$ & $0(0)$ & $2(33.3)$ & $0(0)$ & $0(0)$ \\
\hline Dysgeusia & $2(33.3)$ & $0(0)$ & $0(0)$ & $1(16.7)$ & $0(0)$ & $0(0)$ \\
\hline Hypoesthesia & $2(33.3)$ & $0(0)$ & $0(0)$ & $0(0)$ & $0(0)$ & $0(0)$ \\
\hline Fatigue & $2(33.3)$ & $0(0)$ & $0(0)$ & $4(66.7)$ & $0(0)$ & $0(0)$ \\
\hline Pyrexia & $1(16.7)$ & $0(0)$ & $0(0)$ & $3(50.0)$ & $0(0)$ & $0(0)$ \\
\hline
\end{tabular}

$G$ grade, $G G T$ gamma-glutamyltransferase, ipi ipilimumab

${ }^{a}$ Only toxicities that reached Grade 3/4 in severity or occurred in $>20 \%$ of patients in either cohort are presented

${ }^{\mathrm{b}}$ Assessment based on National Cancer Institute Common Terminology Criteria for Adverse Events version 3.0 
bilirubin, and grade 4 increase in lipase; $n=1$, grade 3 increase in gamma-glutamyltransferase [GGT]). The grade 3 enterocolitis resolved with treatment with a high-dose steroid, and the grade 3 increase in blood bilirubin was treated with agents including oral prednisolone, levofloxacin, and meropenem, and parenteral nutrition. The grade 4 increase in lipase and grade 3 increase in GGT both resolved without treatment. One patient in Cohort B experienced an irAE of grade 2 pneumonitis on Day 126 after receiving 5 cycles of chemotherapy and 3 doses of ipilimumab. The pneumonitis resolved following treatment with intravenous methylprednisolone and then oral prednisolone which was gradually tapered.

\section{Efficacy data}

Efficacy was assessed in patients who received at least one dose of study medication $(N=15)$. In Cohort $\mathrm{A}$ ( $3 \mathrm{mg} / \mathrm{kg}, n=8), 3$ patients achieved a partial response (PR) and an additional 3 patients achieved stable disease (SD). Two patients were not evaluable because they discontinued prior to the first tumor assessment due to chemotherapy toxicity. In Cohort B (10 mg/kg, $n=7), 3$ patients achieved a PR and an additional 4 patients achieved SD. One patient who achieved SD requested to discontinue prior to the first dose of ipilimumab $10 \mathrm{mg} / \mathrm{kg}$.

Table 4 Immune-related adverse events in patients who received PTX/CBDCA and at least one dose of ipilimumab

\begin{tabular}{|c|c|c|c|c|c|c|}
\hline \multirow[t]{2}{*}{ Toxicities $^{\mathrm{a}}$} & \multicolumn{3}{|c|}{$\begin{array}{l}\text { Cohort A } \\
(n=6) \\
\text { Ipi } 3 \mathrm{mg} / \mathrm{kg}\end{array}$} & \multicolumn{3}{|c|}{$\begin{array}{l}\text { Cohort B } \\
(n=6) \\
\text { Ipi } 10 \mathrm{mg} / \mathrm{kg}\end{array}$} \\
\hline & $\mathrm{G} 1 / 2$ & $\mathrm{G} 3 / 4$ & Total & $\mathrm{G} 1 / 2$ & $\mathrm{G} 3 / 4$ & Total \\
\hline Skin and subcutaneous tissue disorders & $5(83.3)$ & $0(0)$ & $5(83.3)$ & $6(100.0)$ & $0(0)$ & $6(100.0)$ \\
\hline Rash & $5(83.3)$ & $0(0)$ & $5(83.3)$ & $3(50.0)$ & $0(0)$ & $3(50.0)$ \\
\hline Alopecia & $1(16.7)$ & $0(0)$ & $1(16.7)$ & $2(33.3)$ & $0(0)$ & $2(33.3)$ \\
\hline Pruritus & $0(0)$ & $0(0)$ & $0(0)$ & $2(33.3)$ & $0(0)$ & $2(33.3)$ \\
\hline Pigmentation disorder & $1(16.7)$ & $0(0)$ & $1(16.7)$ & $0(0)$ & $0(0)$ & $0(0)$ \\
\hline Dermatitis acneiform & $0(0)$ & $0(0)$ & $0(0)$ & $1(16.7)$ & $0(0)$ & $1(16.7)$ \\
\hline Rash maculo-papular & $0(0)$ & $0(0)$ & $0(0)$ & $1(16.7)$ & $0(0)$ & $1(16.7)$ \\
\hline Gastrointestinal disorders & $4(66.7)$ & $0(0)$ & $4(66.7)$ & $1(16.7)$ & $1(16.7)$ & $2(33.3)$ \\
\hline Diarrhea & $3(50.0)$ & $0(0)$ & $3(50.0)$ & $1(16.7)$ & $0(0)$ & $1(16.7)$ \\
\hline Gastritis & $1(16.7)$ & $0(0)$ & $1(16.7)$ & $0(0)$ & $0(0)$ & $0(0)$ \\
\hline Stomatitis & $1(16.7)$ & $0(0)$ & $1(16.7)$ & $1(16.7)$ & $0(0)$ & $1(16.7)$ \\
\hline Enterocolitis & $0(0)$ & $0(0)$ & $0(0)$ & $0(0)$ & $1(16.7)$ & $1(16.7)$ \\
\hline Nervous system disorders & $3(50.0)$ & $0(0)$ & $3(50.0)$ & $5(83.3)$ & $0(0)$ & $5(83.3)$ \\
\hline Hypoesthesia & $2(33.3)$ & $0(0)$ & $2(33.3)$ & $0(0)$ & $0(0)$ & $0(0)$ \\
\hline Peripheral motor neuropathy & $1(16.7)$ & $0(0)$ & $1(16.7)$ & $0(0)$ & $0(0)$ & $0(0)$ \\
\hline Peripheral sensory neuropathy & $1(16.7)$ & $0(0)$ & $1(16.7)$ & $5(83.3)$ & $0(0)$ & $5(83.3)$ \\
\hline Endocrine disorders & $1(16.7)$ & $1(16.7)$ & $2(33.3)$ & $0(0)$ & $0(0)$ & $0(0)$ \\
\hline Adrenal insufficiency & $0(0)$ & $1(16.7)$ & $1(16.7)$ & $0(0)$ & $0(0)$ & $0(0)$ \\
\hline Hypoparathyroidism & $1(16.7)$ & $0(0)$ & $1(16.7)$ & $0(0)$ & $0(0)$ & $0(0)$ \\
\hline Laboratory investigations & $0(0)$ & $1(16.7)$ & $1(16.7)$ & $1(16.7)$ & $2(33.3)$ & $3(50.0)$ \\
\hline Amylase increased & $0(0)$ & $1(16.7)$ & $1(16.7)$ & $1(16.7)$ & $0(0)$ & $1(16.7)$ \\
\hline Blood bilirubin increased & $0(0)$ & $0(0)$ & $0(0)$ & $0(0)$ & $1(16.7)$ & $1(16.7)$ \\
\hline Blood corticotrophin decreased & $0(0)$ & $0(0)$ & $0(0)$ & $1(16.7)$ & $0(0)$ & $1(16.7)$ \\
\hline Blood cortisol decreased & $0(0)$ & $0(0)$ & $0(0)$ & $1(16.7)$ & $0(0)$ & $1(16.7)$ \\
\hline Blood TSH decreased & $0(0)$ & $0(0)$ & $0(0)$ & $1(16.7)$ & $0(0)$ & $1(16.7)$ \\
\hline GGT increased & $0(0)$ & $0(0)$ & $0(0)$ & $0(0)$ & $1(16.7)$ & $1(16.7)$ \\
\hline Lipase increased & $0(0)$ & $0(0)$ & $0(0)$ & $0(0)$ & $1(16.7)$ & $1(16.7)$ \\
\hline Respiratory, thoracic and mediastinal disorders & $0(0)$ & $0(0)$ & $0(0)$ & $1(16.7)$ & $0(0)$ & $1(16.7)$ \\
\hline Pneumonitis & $0(0)$ & $0(0)$ & $0(0)$ & $1(16.7)$ & $0(0)$ & $1(16.7)$ \\
\hline
\end{tabular}

$G$ grade, GGT gamma-glutamyltransferase, ipi ipilimumab, TSH thyroid-stimulating hormone

${ }^{a}$ Assessment based on National Cancer Institute Common Terminology Criteria for Adverse Events version 3.0 
Table 5 Summary statistics for ipilimumab pharmacokinetic parameters during Cycle 3 and data from non-Japanese patients in other studies

\begin{tabular}{|c|c|c|c|c|c|c|}
\hline Dose & $\begin{array}{l}\mathrm{C}_{\max }(\mu \mathrm{g} / \mathrm{mL}) \\
\text { Geo. Mean } \\
(\% \mathrm{CV})\end{array}$ & $\begin{array}{l}\mathrm{AUC}_{(0-21 \mathrm{~d})} \\
(\mu \mathrm{g} \times \mathrm{hr} / \mathrm{mL}) \\
\text { Geo. Mean }(\% \mathrm{CV})\end{array}$ & $\begin{array}{l}\mathrm{T}_{\max }(\mathrm{h}) \\
\text { Median } \\
\text { (Min-Max) }\end{array}$ & $\begin{array}{l}\text { T-HALF (day) } \\
\text { Mean (SD) }\end{array}$ & $\begin{array}{l}\text { CL }(\mathrm{mL} / \mathrm{hr}) \\
\text { Geo. Mean } \\
(\% \mathrm{CV})\end{array}$ & $\begin{array}{l}\text { Vss (L) } \\
\text { Geo. Mean } \\
(\% \mathrm{CV})\end{array}$ \\
\hline \multicolumn{7}{|l|}{ Japanese patients (current study) } \\
\hline Cohort A Ipi 3 mg/kg $(n=6)$ & $72.8(12)$ & $12,632(12)$ & $2.75(1.37-4.08)$ & $13.3(3.64)$ & $12(17)$ & $4.97(17)$ \\
\hline Cohort B Ipi $10 \mathrm{mg} / \mathrm{kg}(n=6)$ & $201(21)$ & $36,489(21)$ & $3.98(1.45-23.8)$ & $11.3(2.83)$ & $14.4(25)$ & $5.35(22)$ \\
\hline \multicolumn{7}{|c|}{ Non-Japanese patients (historical data) } \\
\hline Ipi $3 \mathrm{mg} / \mathrm{kg}(N=12)^{\mathrm{a}}$ & 84.5 & 12,384 & N/A & 15.25 & $\mathrm{~N} / \mathrm{A}$ & N/A \\
\hline Ipi $10 \mathrm{mg} / \mathrm{kg}(N=14)^{\mathrm{b}}$ & $234.54(29)$ & $46925.36(36)$ & $1.51(1.5-24.0)$ & $13.93(7.54)$ & $11.63(50)$ & $5.10(25)$ \\
\hline
\end{tabular}

$A U C$ area under the concentration-versus-time curve, $C L$ clearance, $C_{\max }$ peak plasma concentration, $C V$ coefficient of variation, ipi ipilimumab, $N / A$ data not available, $S D$ standard deviation, $T-H A L F$ time required to decrease the drug concentration in the blood by half, VSS volume of distribution at steady-state

${ }^{a}$ Data from non-Japanese melanoma patients treated with hybridoma-derived ipilimumab $3 \mathrm{mg} / \mathrm{kg}$ monotherapy in study MDX010-15 [9]

${ }^{\mathrm{b}}$ Data from non-Japanese melanoma patients treated with ipilimumab $10 \mathrm{mg} / \mathrm{kg}$ plus paclitaxel and carboplatin in study CA184-078 [10]

\section{Pharmacokinetic data}

Table 5 provides summary statistics for ipilimumab PK parameters during Cycle 3 and PK data from non-Japanese patients treated with ipilimumab in previous clinical studies [9, 10]. Serum ipilimumab levels were elevated with median values of $\mathrm{T}_{\max }$ from 2.75 to $3.98 \mathrm{~h}$. The observed mean $\mathrm{T}$ HALF of ipilimumab was approximately 11 to 13 days. The geometric mean CL was 12.0 to $14.4 \mathrm{~mL} / \mathrm{h}$, and Vss was approximately $5 \mathrm{~L}$. The PK profile in this study was similar to that reported in previous clinical trials of non-Japanese patients $[9,10]$. The concentration of serum ipilimumab over time is shown in Fig. 2.

\section{Immunogenicity}

A total of 61 samples from the 12 ipilimumab-treated patients were evaluated for the presence of HAHA to ipilimumab; all samples were negative for HAHA.

\section{Discussion}

In this phase I study in Japanese patients with advanced NSCLC using the definition defined in the protocol, and agreed upon by the sponsor and $\mathrm{Pl}$, the recommended dose was identified as $10 \mathrm{mg} / \mathrm{kg}$ of ipilimumab in a phased combination with PTX/CBDCA. This dosage of ipilimumab is being studied in two on-going phase III lung cancer studies: CA184-104 (CT.gov, NCT01285609) and CA184-156 (CT.gov, NCT01450761) [11, 12].

Overall, the safety profile was consistent with data from previous studies of ipilimumab in combination with PTX/ CBDCA [6] and known toxicities of ipilimumab monotherapy [13] and PTX and CBDCA [14]. Two out of 6 patients treated with ipilimumab $3 \mathrm{mg} / \mathrm{kg}$ experienced DLTs with 3 events reported: grade 3 febrile neutropenia, grade 4 increased amylase, and grade 4 thrombocytopenia. The increased amylase required no treatment. Transient febrile neutropenia and thrombocytopenia are common chemotherapy-related AEs
Fig. 2 Ipilimumab serum concentration profile versus time during Cycle 3. Open circles, ipilimumab $3 \mathrm{mg} / \mathrm{kg}$ plus PTX/CBDCA group $(n=6)$; closed circles, ipilimumab $10 \mathrm{mg} / \mathrm{kg}$ plus PTX/CBDCA group $(n=6)$. Data are mean plus standard deviation. On Day 1 of Cycle 3, blood serum samples were drawn at the following time points: Pre-infusion, $1.5 \mathrm{~h}$ (just prior to the end of ipilimumab dosing), $4 \mathrm{~h}$ and $24 \mathrm{~h}$

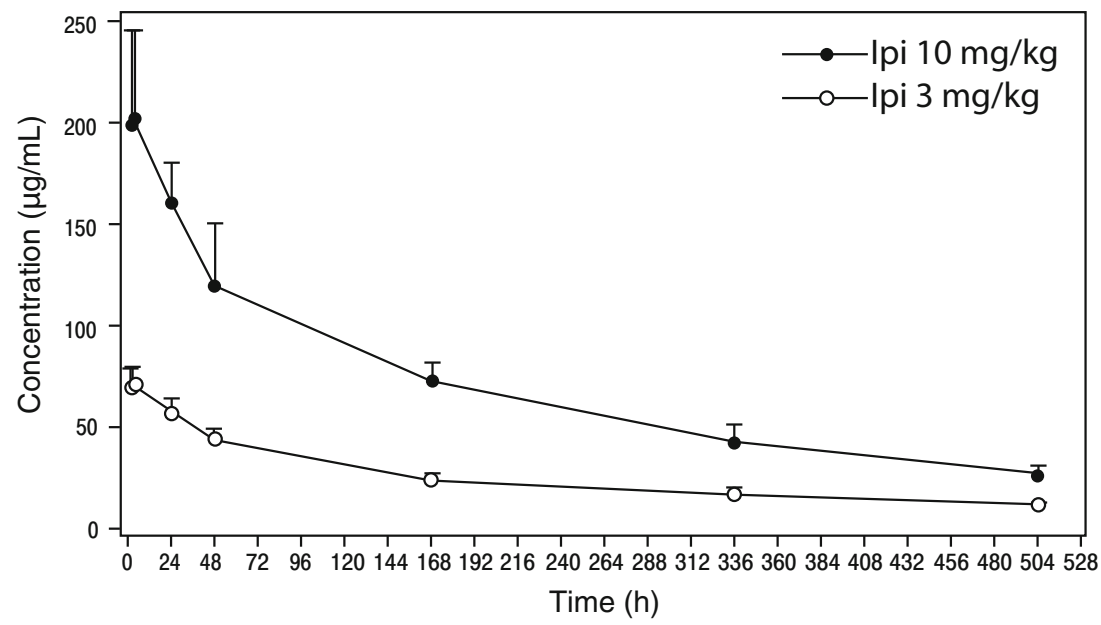


[14]. One of 6 patients treated with ipilimumab $10 \mathrm{mg} / \mathrm{kg}$ had DLT with 3 events reported: grade 3 enterocolitis, grade 3 increased total bilirubin, and grade 4 increased lipase. Enterocolitis is a common irAE associated with ipilimumab [13]. Increased bilirubin has been described as associated with ipilimumab [13] and chemotherapy [15], and increased lipase is commonly experienced as a chemotherapy-related $\mathrm{AE}$ [16] as well. In both dose cohorts, the majority of AEs were hematological, although non-hematological toxicities were also reported (Table 3). There was no clear association between the incidence of AEs and the dose of ipilimumab, as reported in prior studies [17]. Most common safety events associated with ipilimumab are inflammatory in nature, which may reflect its immune-based mechanism of action [18]. The most common irAEs across the two study cohorts were grade $1 / 2$ toxicities affecting the skin, gastrointestinal tract, and nervous system. Immune-related toxicities were manageable with established treatment guidelines [13].

Although conclusions regarding efficacy in phase I studies are necessarily limited, ipilimumab in a phased combination with PTX and CBDCA demonstrated encouraging preliminary antitumor activity. Among the 12 evaluable patients who received at least one dose of ipilimumab, 3 patients in each cohort achieved a partial response, and an additional 3 patients in each cohort achieved stable disease. Stable disease is a potentially clinically relevant outcome with ipilimumab treatment because some patients experience a slow and steady decrease in total tumor burden following a period of SD [19, 20]. Antitumor activity was demonstrated also in the phase II CA184-041 study [6]. Among patients who received phased ipilimumab in combination with PTX and CBDCA $(n=68)$ in study CA184-041, 22 achieved an immune-related partial response and 37 had an immune-related SD, as assessed by a blinded independent radiologic review committee $[6,20]$.

The PK profile of ipilimumab at two doses, $3 \mathrm{mg} / \mathrm{kg}$ and $10 \mathrm{mg} / \mathrm{kg}$, was also evaluated in the current study. Data from previous studies have shown no major PK interactions between ipilimumab administered at $10 \mathrm{mg} / \mathrm{kg}$ in Caucasians and PTX/ CBDCA [10]. Acknowledging the limitations of comparing data from different studies, the PK characteristics of ipilimumab observed in the current study were similar to those previously reported in clinical trials of non-Japanese patients $[9,10]$.

Although ipilimumab is a fully human antibody, it has the potential to induce formation of anti-drug antibodies when it is administered as a therapeutic agent [21]. None of the ipilimumab-treated patients in this study had HAHA, and therefore there was no evidence of an anti-ipilimumab antibody response.

In summary, the recommended dose for ipilimumab in combination with PTX/CBDCA in Japanese patients with NSCLC was identified as $10 \mathrm{mg} / \mathrm{kg}$. The first 2 treatment cycles in this study were PTX/CBDCA every 3 weeks and the last 4 treatment cycles were ipilimumab plus PTX/
CBDCA every 3 weeks. The safety profile of ipilimumab in combination with PTX and CBDCA was consistent with the previously defined profile established in global clinical studies. Overall, the PK characteristics of ipilimumab in combination with PTX and CBDCA were similar to those demonstrated in global clinical studies in non-Japanese subjects.

Acknowledgments We would like to thank the patients and investigators who participated in study CA184-113. This study was funded by Bristol-Myers K.K. The authors wish to acknowledge Jennifer DiNieri and Karin McGlynn of StemScientific, an Ashfield Company, part of UDG Healthcare plc, funded by Bristol-Myers Squibb, for providing writing and editorial support.

\section{Ethical statement}

Conflicts of interest H. Horinouchi has received research grants from the National Cancer Center Research and Development fund and honoraria for speaking at symposia from Johnson \& Johnson, TAIHO Pharmaceuticals, and Eli Lilly. N. Yamamoto has received research funding from Bristol-Myers Squibb. T. Tokudome is an employee of Bristol-Myers K.K. S. Yagishita, H. Wakui, S. Kitazono, H. Mizugaki, S. Kanda, Y. Fujiwara, H. Nokihara, I. Sekine, K. Kubota, and T. Tamura report no conflicts of interest.

Research involving human participants The study protocol was approved by the Institutional Review Board and research was conducted in accordance with the standards specified by Article 14 Paragraph 3 and Article 80-2 of the Pharmaceutical Affairs Law, and Good Clinical Practice, as defined by the Ministerial Ordinance Concerning the Standards for the Implementation of Clinical Studies on Pharmaceutical Products and concerning notifications.

Informed consent All study participants provided written informed consent prior to enrollment.

Open Access This article is distributed under the terms of the Creative Commons Attribution 4.0 International License (http:// creativecommons.org/licenses/by/4.0/), which permits unrestricted use, distribution, and reproduction in any medium, provided you give appropriate credit to the original author(s) and the source, provide a link to the Creative Commons license, and indicate if changes were made.

\section{References}

1. National Comprehensive Cancer Network (2014) Clinical practice guidelines in oncology: small cell lung cancer (version 1.2015). http://www.nccn.org/professionals/physician_gls/pdf/sclc.pdf. Accessed 10 Nov 2014

2. Brahmer JR, Pardoll DM (2013) Immune checkpoint inhibitors: making immunotherapy a reality for the treatment of lung cancer. Cancer Immunol Res 1(2):85-91

3. Hoos A, Ibrahim R, Korman A, Abdallah K, Berman D, Shahabi V, Chin K, Canetta R, Humphrey R (2010) Development of ipilimumab: contribution to a new paradigm for cancer immunotherapy. Semin Oncol 37(5):533-546

4. Hanaizi Z, van Zwieten-Boot B, Calvo G, Lopez AS, van Dartel M, Camarero J, Abadie E, Pignatti F (2012) The European Medicines Agency review of ipilimumab (Yervoy) for the treatment of advanced (unresectable or metastatic) melanoma in adults who have received prior therapy: summary of the scientific assessment of the 
Committee for Medicinal Products for Human Use. Eur J Cancer 48(2):237-242

5. Pazdur R (2013) FDA Approval for Ipilimumab. National Cancer Institute at the National Institutes of Health. http://www.cancer.gov/ cancertopics/druginfo/fda-ipilimumab. Accessed 19 Aug 2014

6. Lynch TJ, Bondarenko I, Luft A, Serwatowski P, Barlesi F, Chacko R, Sebastian M, Neal J, Lu H, Cuillerot JM, Reck M (2012) Ipilimumab in combination with paclitaxel and carboplatin as first-line treatment in stage IIIB/IV non-small-cell lung cancer: results from a randomized, double-blind, multicenter phase II study. J Clin Oncol 30(17):2046-2054

7. Cancer Therapy Evaluation Program (2006) Common terminology criteria for adverse events (version 4.0). http://evs.nci.nih.gov/ftp1/ CTCAE/About.html. Accessed 9 Feb 2015

8. Eisenhauer EA, Therasse P, Bogaerts J, Schwartz LH, Sargent D, Ford R, Dancey J, Arbuck S, Gwyther S, Mooney M, Rubinstein L, Shankar L, Dodd L, Kaplan R, Lacombe D, Verweij J (2009) New response evaluation criteria in solid tumours: revised RECIST guideline (version 1.1). Eur J Cancer 45(2):228-247

9. Weber JS, O’Day S, Urba W, Powderly J, Nichol G, Yellin M, Snively J, Hersh E (2008) Phase I/II study of ipilimumab for patients with metastatic melanoma. J Clin Oncol 26(36):5950-5956

10. Weber J, Hamid O, Amin A, O'Day S, Masson E, Goldberg SM, Williams D, Parker SM, Chasalow SD, Alaparthy S, Wolchok JD (2013) Randomized phase I pharmacokinetic study of ipilimumab with or without one of two different chemotherapy regimens in patients with untreated advanced melanoma. Cancer Immun 13:7

11. Clinicaltrials.gov (2011) Randomized, Multicenter, Double-Blind, Phase 3 Trial Comparing the Efficacy of Ipilimumab in Addition to Paclitaxel and Carboplatin Versus Placebo in Addition to Paclitaxel and Carboplatin in Subjects With Stage IV/Recurrent Non Small Cell Lung Cancer (NSCLC) http://clinicaltrials.gov/ct2/show/ NCT01285609. Accessed 19 Aug 2014

12. Clinicaltrials.gov (2011) Randomized, Multicenter, Double-Blind, Phase 3 Trial Comparing the Efficacy of Ipilimumab Plus Etoposide/Platinum Versus Etoposide/Platinum in Subjects With
Newly Diagnosed Extensive-Stage Disease Small Cell Lung Cancer (ED-SCLC) http://clinicaltrials.gov/ct2/show/NCT01450761. Accessed 19 Aug 2014

13. Bristol-Myers Squibb (2011) Yervoy (ipilimumab) Risk Evaluation and Mitigation Strategy (REMS): Immune-mediated adverse reaction management guide. https://www.hcp.yervoy.com/pages/rems. aspx. Accessed 19 Aug 2014

14. Loprinzi CL, Messner C (2012) Understanding and managing chemotherapy side effects. Cancercare Connect booklet series. http:// www.cancercare.org/publications/24-understanding_and_ managing_chemotherapy_side_effect. Accessed 19 Aug 2014

15. King PD, Perry MC (2001) Hepatotoxicity of chemotherapy. Oncologist 6(2):162-176

16. Chemocare.com (2015) Hyperlipasemia (high blood lipase level). $\mathrm{http} / / /$ chemocare.com/chemotherapy/side-effects/hyperlipasemiahigh-lipase.aspx\#.U2zxEvldXxS. Accessed 19 Aug 192014

17. Feng Y, Roy A, Masson E et al (2013) Exposure-response relationships of the efficacy and safety of ipilimumab in patients with advanced melanoma. Clin Cancer Res 19: 3977-3986

18. Wolchok JD, Hodi FS, Weber JS, Allison JP, Urba WJ, Robert C, O’Day SJ, Hoos A, Humphrey R, Berman DM, Lonberg N, Korman AJ (2013) Development of ipilimumab: a novel immunotherapeutic approach for the treatment of advanced melanoma. Ann N Y Acad Sci 1291:1-13

19. Hoos A (2012) Evolution of end points for cancer immunotherapy trials. Ann Oncol 23(Suppl 8):viii47-52

20. Wolchok JD, Hoos A, O'Day S, Weber JS, Hamid O, Lebbé C, Maio M, Binder M, Bohnsack O, Nichol G, Humphrey R, Hodi FS (2009) Guidelines for the evaluation of immune therapy activity in solid tumors: immune-related response criteria. Clin Cancer Res 15(23):7412-7420

21. Brennan FR, Morton LD, Spindeldreher S, Kiessling A, Allenspach R, Hey A, Muller PY, Frings W, Sims J (2010) Safety and immunotoxicity assessment of immunomodulatory monoclonal antibodies. MAbs 2(3):233-255 